\title{
A study on surveillance of vaccine preventable disease and adverse events following immunization
}

\author{
Vijaydeep Siddharth ${ }^{1, *}$, Vivek Adhish ${ }^{2}$, J. K. Dass ${ }^{3}$ \\ ${ }^{1}$ Assistant Professor, Dept. of Hospital Administration, All India Institute of Medical Sciences, New Delhi, ${ }^{2}$ Professor, Dept. of \\ Community Health Administration, ${ }^{3}$ Director, National Institute of Health \& Family Welfare, New Delhi, India
}

*Corresponding Author:

Email: dr.siddharthmamc@gmail.com

\begin{abstract}
Introduction: The surveillance of public health problem is an important task in public health. It is essential to include Vaccine Preventable Diseases (VPD) surveillance in Universal Immunisation Programme (UIP) in order to know its impact-

Aims: The present study was an attempt to examine the various aspects of existing surveillance system for vaccine preventable diseases and adverse event following immunization in Ghaziabad district of UP state in India.

Materials and Methods: This study was descriptive and observational in nature, and was carried out in Ghaziabad district of Uttar Pradesh, India for a period of four months from $1^{\text {st }}$ November 2008 to $28^{\text {th }}$ February 2009. Dasna block in the district was selected through simple random sampling and all the Primary Health Centres and Sub-centres were studied.

Results: Study findings revealed that surveillance data on VPDs and AEFI is obtained mainly through routine reporting. More than $50 \%$ children immunized by $70 \%$ ANM reported back with complication. Once a VPD case and death is reported the preventive, therapeutic, anti epidemic measures and health camps are organised.

Conclusions: Sincere and sustained efforts are needed for implementation of surveillance programme for VPDs and AEFI as current picture is not very encouraging.

Keywords: Epidemiology, Surveillance, Vaccine Preventable Diseases (VPD), Adverse Events Following Immunization (AEFI) Key messages: The manpower and infrastructure are usually in place, despite that the implementation of the surveillance of VPDs and AFEIs is lacking in different healthcare facilities. More stress should be laid on the training of the healthcare personnel in surveillance of VPDs and AEFIs, supervision and monitoring.
\end{abstract}

\section{Introduction}

Surveillance is the foundation of sound public health practice; however, disease surveillance systems are often fragmented and vertical, based on the characteristics of the targeted disease or syndrome, and the characteristics of the existing public health infrastructure. ${ }^{1}$ The surveillance of public health problem is an important task in public health. ${ }^{2}$ It is essential to include Vaccine Preventable Diseases (VPD) surveillance in Universal Immunisation Programme (UIP) in order to know its impact. Ten major attributes of a VPD surveillance system include the existence and use of case definitions, a case detection system, a process for case notification, procedures for case investigation, including standardized data variables, data management procedures, including data analysis and information reporting, outbreak response guidelines, laboratory algorithms and standard procedures, final classification procedures, feedback to partners and clear program management and supervision. ${ }^{1}$

Vaccines can cause minor and serious adverse events, therefore, it is extremely important that Adverse Event Following Immunisation (AEFI) are reported, investigated and treated, as it will help to improve the quality and in long run boost public confidence in the programme. The final output of the surveillance system often takes the shape of a report to the decision makers. The use to which the report will be put is the ultimate test of whether the surveillance system works. Such surveillance systems can monitor feedback and improvements that takes place in response to analyzing and interpreting the data. ${ }^{3}$ It was aimed to study is an attempt to examine the operational aspects of existing surveillance system for VPDs and AEFIs at the district level as it acts as a nodal independent entity for programme implementation.

\section{Materials and Methods}

This descriptive and observational study was designed to study surveillance of VPDs and AEFIs. It was carried out over a period of four months i.e. from November 2008 to February 2009 in Uttar Pradesh (UP) state of India. Ghaziabad district of UP was selected through purposive sampling considering time and resources constraint. Surveillance system of VPDs and AEFIs was studied at District level, Community Health Centre (CHC), Primary Health Centre (PHC) and Sub centre (SC) level. One CHC in Ghaziabad district was selected through simple random sampling and all the PHCs, SCs under its jurisdiction were included for study. Surveillance system of VPDs and AEFIs was studied by conducting structured interview, review of records and field visits. Structured interview were held with Chief Medical Officer (CMO), District Immunization officer (DIO), Medical Officer i/c's of respective areas, Lady Health Visitor (LHV), and Auxiliary Nurse Midwife (ANM) in the study area. Pretesting of the interview tool was done on a sample of 
$10 \%$ and was modified accordingly Data was analyzed using Microsoft excel software.

\section{Results}

Study was carried out in the purposively selected Ghaziabad District which is located in the western part of Uttar Pradesh state of India. There are 8 blocks in Ghaziabad district and CHC of Dasna block was selected through simple random sampling. CHC Dasna is located approximately $12 \mathrm{kms}$ from Ghaziabad city and has got 4 PHCs working under it. Dasna block is having rural population predominantly. In total there are 32 sub centres working under the CHC Dasna. $\mathrm{CMO}$ and DIO were interviewed at the district level. At CHC level, there is Block Level Immunization Officer, three lady health visitors and 35 ANMs who were interviewed.

\section{Organisational Set Up}

At central level and state level, Mission Director, National Rural Health Mission (NRHM) and State Expanded Programme for Immunization (EPI) Officer are responsible for overall implementation of Universal Immunization Programme (UIP) including surveillance of VPDs and AEFIs. Regional Investigation Teams primarily investigate serious AEFIs and deaths (investigation will be initiated within 24hours of being reported to the team).

At the district level CMO is responsible for overall implementation of UIP including surveillance of VPDs and AEFIs. The CMO has delegated this function to DIO who is of the rank of Additional CMO. He is assisted by a clerk and a statistical assistant. At the block and PHC level, Medical Officer in Charge is responsible for implementation of UIP including surveillance of VPDs and AEFIs respectively. MO I/C, CHC who is of the rank of Deputy CMO is assisted by an Immunization Officer and ICC (investigator cum clerk) in his office. At sub centre level ANM is responsible for immunization and surveillance activities.

DIO's is responsible for training, motivation of district and PHC staff in surveillance of VPDs and AEFIs, verification of VPD cases and deaths. MO I/C, PHC is mainly responsible for verification of VPD cases, collection and compilation of VPD reports. Health workers and health supervisors were responsible for collection and reporting of VPDs in their areas as well as maintenance of data related to VPDs.

At district level there is availability of computer, internet connection, telephone, stationery but at block level these facilities are lacking except for poorly functioning telephone line. At the PHC, above mentioned facilities are lacking.

\section{Knowledge and Awareness}

Majority of health worker were able to tell the diseases are prevented through routine immunization while only $40 \%$ ANMs were able to describe symptoms of all 6 VPDs. Many of them felt the need of practical training/skill based training for identification of VPDs.

Health worker were able to enumerate the adverse events following DPT and BCG vaccines but with regard to other vaccines they had no knowledge. They brought out the fact that complications of fever and swelling increase the drop out in immunization. How significantly it is adding to drop out cannot be commented.

\section{Ability to identify VPD}

Only knowledge component related to identification of VPD was assessed. The knowledge component needs to be increased as only half of the ANM/LHV expressed that they can confidently identify VPDs and enumerate the signs and symptoms.

Almost $55 \%$ of the health workers were able to describe the symptoms of different VPDs and only 5\% were not familiar with the symptoms of VPDs. Rest were able to tell the symptoms of less than 3 VPDs. All the LHVs could identify VPD.

From the analysis it was found that educational level has a bearing on identification of VPDs but whether with increase in education status improves the ability to identify VPDs could not be statistically confirmed. Further research is required to substantiate it. Only 30\% of health workers have received any sort of material to increase their knowledge with regards to VPDs and booklets (80\%) was the most commonly used educational tool.

\section{Percentage of children reporting back with complication}

More than $50 \%$ children immunized by $70 \%$ ANM reported back with complication like fever, swelling, and redness which were commonly associated with DPT immunization. All the health workers stated that it is important to collect data on VPDs but none was reporting in the provided formats and were verbally communicating information occurrence of VPDs to MO I/C of their respective area. Most of the health workers were neither sending reports nor maintaining records of VPDs and AEFIs.

\section{Supervision}

At the field / sub center level the immunization and surveillance work of health workers was supervised by LHV by making field visits, spot verification, verification of vaccination coverage register, monthly report and visiting the houses of the reported cases. The activities of the LHV in turn are supervised by the medical officer by record verification and field visits. At the block level MO I/C supervise the work of all LHVs and medical officers through record verification checking. No punitive action is taken against erring staff except issue of warning and memorandum. CMO 
and DIO supervises the surveillance work through records review and making field visits.

\section{Training status}

$\mathrm{CMO}$ and DIO have received training in routine immunization and IDSP. All MO have received training in Routine Immunization (5 Days) which was conducted at District hospital, Ghaziabad by Regional Medical College. None of the MO has received training under IDSP.

Every ANM in the district has received basic training at ANMTCs. $40 \%$ of health worker have not received any training on immunization. As such there is no training policy at the district or block level but only hands on training is administered at monthly meeting of ANM. No hands on or orientation training has been imparted to health workers in past one year. None of the health workers have neither received any training on surveillance of VPDs and AEFIs nor were familiar with the concept of surveillance of VPDs. On analysis it was found that health workers who have received training in immunization have better knowledge with regards to knowledge of VPD. But to establish this relationship further research is required.

\section{Reporting System}

Routine reporting is done through "Monthly District Performance Report". It has information on Vaccination coverage, Vaccine supply in doses, AD syringes supply, Surveillance of VPD, Untoward reaction, Status of district cold chain equipment. The information from this format needs to be fed in the RIMS software. CHC receives blank format with regards to VPD and AEFI reporting. Blank column are nil reported in the block performance report sent by the MO I/C. CHC receives all the report by $20^{\text {th }}$ of every month. Reports from different $\mathrm{CHC}$ are received at the district level and are compiled by $28^{\text {th }}$ of every month. Then this compiled report is sent to state authorities and then to Assistant Commissioner, Immunization. There is no concept of zero reporting in the district.

Surveillance data on VPDs and AEFI is obtained mainly through routine reporting in addition National Polio Surveillance Project also carries out the surveillance of AFP. Some of the measles cases are being reported under the IDSP. Under IDSP there are 5 reporting units. No IDSP related activities are being carried out in the block; they have only received the guidelines.

There are no reported cases of any irritability, seizures or any other event which is incidental or associated with immunization. No deaths have been reported by any ANM during their entire duration of job in immunization programme. Deep down under there is fear among the ANM regarding the reporting of these AEFI, that it may affect their career. Fever along with swelling is the most common complication following immunization especially after DPT immunization. Other complications like redness, fever, swelling, fever along with redness are also reported. By going through the district monthly reports from year 2004 to 2009, it is very much clear no reporting of AEFI is being done in the district. Even there is no record of AEFI with the field workers.

The health worker collects information on cases of VPDs when either patient visits the sub center for consultation or treatment or during routine domiciliary visit. Cases are also reported by ASHA, AWW and popular people of the village. Then the information is sent to MO verbally with no maintenance of the record of the same. No reporting or record is maintained for AEFI. It can be concluded there is no well defined protocol with regards to reporting of VPDs. All the healthcare workers are reporting cases of VPD, if any, as per their convenience.

\section{Verification Procedure}

Confirmation of VPD is done by medical officer and is done by history, clinical features and physical examination. Sometimes if there is a suspected outbreak then DIO forms a team which visits the area and verifies and confirms the cases. He also issues instructions for follow up action to be taken when cases are reported. $22 \%$ of the neonatal deaths are being verified and not only this but majority of these deaths are being verified by ANM/LHV themselves. Cases of VPD are being verified on reporting by concerned medical officer. Verification of poliomyelitis is done by WHO officials i.e. Surveillance Medical Officer and sometimes by the medical officer of the area. $82.9 \%$ (29 out of 35) of the health worker reported that there were no lame children in their area. Considering the number of polio cases i.e. 33 in 2008-09 in Ghaziabad district and Dasna being high risk area percentage of ANM reporting cases of AFP seems to be small. There was not even a single case of AFP which was not covered or looked after by either SMO or MO incharge. So this shows active surveillance is working in the area for poliomyelitis.

\section{Action taken when VPD cases and deaths are reported}

Once a VPD is reported the preventive, treatment, anti epidemic measures and health camps are organised. Immunization of unimmunized children is carried out to control the spread. This fact was confirmed by $70 \%$ of the ANM. Treatment of the cases is taken care off. At block level, once a VPD is reported then a team consisting of Mo I/C, ANM, MPW, ASHA, AWW, Health Worker, and pradhan goes to the area for verification.

If any suspected VPD deaths occur then, a team comprising of DIO, pediatrician and other officials is formed. This team confirms whether death is due to VPD or due to some other cause by enquiring the parents and studying relevant hospitalization documents 
if any. After the diagnosis is confirmed then active search for cases is carried out, treatment, vaccination, health camps, health education, IEC activities are carried out in the community. As per the DIO no death due to any VPD is reported in past 1 year. As such no record was available at the block level and district level to verify the same.

\section{Action following reporting of AEFI}

As such no AEFI are reported and therefore no action is taken. This fact was confirmed from the CMO as well as record analysis. Neither VPD surveys nor spot map preparation is carried out in the district.

\section{Discussion}

According to the existing set up in India, the surveillance of vaccine preventable diseases is heavily, dependent on the routine reporting of selected items of the VPDs. It was disheartening to note that, in district surveillance of VPD was paid very little importance. During the review of immunisation programme in district Bharuch (Gujarat) in 1987 it was found that surveillance was paid little attention at the district. ${ }^{4}$ In another review of immunisation programme in district Kota (Rajasthan) disease surveillance was found to be practically not existing at PHC level. ${ }^{8}$

The review of UIP conducted by the government of India in the year 2002, in six states namely Uttar Pradesh, Orissa, Bihar, Jharkhand, Madhya Pradesh and Rajasthan, it was found that there is very limited reporting of VPD in these states except for the AFP reporting and VPD surveillance in place is inadequate to assess the impact of UIP. ${ }^{5}$ During comprehensive evaluation of national immunisation programme in Egypt in 1984, regarding surveillance for VPDs it was found that many centers either did not report or under reported EPI diseases. ${ }^{6}$

With regards to logistics, there are adequate vaccine supplies and very rarely there are any stock outs. There is sufficient supply of syringes and stationery. During the National Review of UIP it was found that there is adequate supply of vaccines and stock is well maintained but need is felt to improve logistics system and facilitate alternate vaccine delivery to ANM. ${ }^{5}$

Most of the health workers were not fully aware of the signs and symptoms of VPDs and many were not equipped with the standard case definitions of VPDs. During the review of UIP in district Sagar of Madhya Pradesh, it was observed that most of the staff was unaware of Signs and Symptoms of VPDs. ${ }^{7}$

Many of them felt the lack of practical training or skill based training for identification of VPDs and AEFIs. In the review of universal immunization programme, conducted by Government of India in the state of Uttar Pradesh in the year 2002, it was found that no immunization training is conducted in the state for more than 10 years It was recommended to train all providers, including private sector and NGO, on service delivery. To create district level trainers who can train on all aspects of UIP with practical, hands on training followed by refreshers and supervisory support. As such no specific mention was made on training related to surveillance. ${ }^{5}$

$\mathrm{CMO}$, DIO and one $\mathrm{MO}$ of $\mathrm{CHC}$ have received training in IDSP. Majority of health worker have not received any immunization training. They have only received Pulse polio immunization training during the rounds. In review of immunisation programme in district $\operatorname{Kota}^{8}$ (Rajasthan) it was found that there was need for training of health workers to identify and report VPDs. National review of the UIP, conducted in year 2002, has also stressed on the fact that staff should be trained during regular (weekly and monthly) meetings and "on the job" training using supportive supervision. ${ }^{5}$

Since, 1998 recording and reporting of immunization data has been integrated into broader $\mathrm{RCH}$ formats. This had a negative effect on the responsiveness and quality of immunization data available at the national level. ${ }^{9}$ AEFI are not being reported likely due to apprehension among staff that reporting might invite administrative and punitive action. Health workers did not consider reporting of AEFI as one of their duty. Also during the National Review of UIP it was found that AEFI are not being reported. ${ }^{5}$ Fear to report because of reprisals from higher authorities and lack of knowledge and/or motivation to report VPD/AEFI.

Good quality data was lacking. It was also found during the National Review of UIP that data generated is of poor quality and also data is not used to improve programme. ${ }^{5}$ Records were properly maintained at the district but at sub centre, PHC and $\mathrm{CHC}$ no records were available. No information on neonatal deaths was maintained by ANM/LHV. Also during the review of UIP, in the Uttar Pradesh, weak record keeping and reporting was highlighted. Also in Multi year plan priority action, recording and reporting is mentioned as one of the key areas where action is required and validation of reported data is given due importance.

Private practitioners and local doctors contribute to $80 \%$ of the total hospital attendance. Nothing was done in the district to bring them into the system of surveillance of VPDs and AEFI. As per the National UIP review Report 2002, it was found that neither private practitioner are reporting nor coordinating with government machinery. So government has laid stress on integration of private players by providing them with necessary training, formats and involving them in planning. It is recommended that there is a need to review system to reduce reporting burden and increase value. Also there is need to improve VPD reporting. Health staff should be provided with tools to register, track, monitor, and analyse data. There is also need of validating reported coverage data. ${ }^{5}$ 


\section{Conclusion}

To conclude adequate manpower is in place but infrastructure still needs to be improved below district level for seamless exchange of information and to ensure prompt action in case of occurrence of VPD and AEFI. Health workers are very hard working and dedicated but there is need to increase their knowledge and develop skills by administering proper training, hands on training, providing IEC material. There is should be training policy at each level for providing timely training to health workers. There is need of supportive supervision to allay the fear associated with reporting of VPD and AEFI.

In short we have to work hard to develop a system for surveillance of VPD and AEFI which is reliable, efficient and effective, which will help public health manager to take prompt and timely decision.

\section{References}

1. Hyde TB, Andrus JK, Dietz VJ, Lee CE, Widdowson MA, Verani JR, et al. Critical issues in implementing a national integrated all-vaccine preventable disease surveillance system. Vaccine. 2013 Jul 2 [cited 2014 Feb 26];31 Suppl 3:C94-8. Available from: http://www.ncbi.nlm.nih.gov/pubmed/23777699

2. Wallace B. Robert, Last M. John, Kohatsus Neal, Brownson, Schectec, Switchfield, Zaza. Maxcy-RosenauLast book on public health and preventive medicine: 15 th edition: page no 8 .

3. Magnus M. Essentials of Infectious Diseases Epidemiology. 2008: Page no.165-73.

4. Government of India, "Universal Immunisation Programme National Review, District Bharuch, (Gujarat)", Ministry of Health and Family Welfare, 1987.

5. Ministry of Health and Family Welfare, Universal Immunization programme review. Year 2002.

6. Immunising the World's Children - Population report series L Number 5, March, April 1986.

7. Government of India "Universal Immunisation Programme -National Review, District Sagar (Madhya Pradesh)", Ministry of Health and Family, Welfare, 1987.

8. Government of India, National Review on Universal Immunisation Programme, District Kota (Rajasthan) In joint collaboration, Directorate of Medical Health and Family welfare, Rajasthan and Union Ministry of Health and Family Welfare, 1987.

9. WHO. WHO strategic plan for strengthening routine immunization in India. April 2004 - March 2007. Page no. 10 